\title{
Prospective intra/inter-observer evaluation of pre-brachytherapy cervical cancer tumor width measured in TRUS and MR imaging
}

\author{
Mario Federico ${ }^{1 *}$ (D), Carmen Rosa Hernandez-Socorro², Ivone Ribeiro', Jesus Gonzalez Martin³, \\ Maria Dolores Rey-Baltar Oramas ${ }^{1}$, Marta Lloret Saez-Bravo ${ }^{1}$ and Pedro Carlos Lara Jimenez ${ }^{1}$
}

\begin{abstract}
Background: Ultrasound (US) imaging has been proved as an excellent diagnostic tool in gynecology and, due to its wide availability and limited cost, is under intense investigation as base for dose adaptation in cervical cancer brachytherapy. Purpose of this work is to test inter/intra-observer uncertainties between magnetic resonance (MR) and trans-rectal ultrasound (TRUS) imaging in defining maximum tumor width before first brachytherapy (BT) application in a prospective cohort of cervical cancer patients undergoing image-guided adaptive brachytherapy (IGABT).
\end{abstract}

Methods: One hundred ten consecutive cervical cancer patients treated between 2013 and 2016 were included. Before the first BT implant patients underwent MR and TRUS scan with no applicator in place. Images were independently analyzed by three examiners, blinded to the other's results. With clinical information at hand, maximum tumor width was measured on preBT TRUS and MR. Quantitative agreement analysis was undertaken. Intra-class correlation coefficient (ICC), Passing-Bablok and Bland Altman plots were used to evaluate the intra/interobservers measurement agreement.

Results: Average difference between tumor width measured on MR (HRCTV $\mathrm{MR}_{\text {R }}$ ) and TRUS (HRCTV TRUS) was $1.3 \pm 3.2$ $\mathrm{mm}(p<0.001) ; 1.1 \pm 4.6 \mathrm{~mm}(p=0.01)$ and $0.7 \pm 3 \mathrm{~mm}(p=0.01)$. The error was less than $3 \mathrm{~mm}$ in 79,82 and $80 \%$ of the measurements for the three observers, respectively. Intra-observer ICC was 0.96 (C195\% 0.94-0.97), 0.93

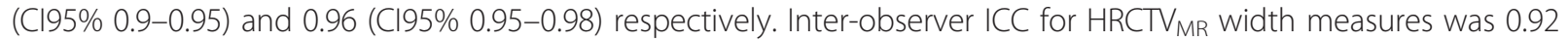
(Cl95\% 0.89-0.94) with no difference among FIGO stages. Inter-observer ICC for HRCTV TRUS was 0.86 (C195\% 0.810.9). For FIGO stage I and II tumors, ICC HRCTV TRUS $_{\text {values were comparable to respective HRCTV }}$ MR ICC values. For larger tumors HRCTV TRUS inter-observer ICC values were lower than respective HRCTV $V_{M R}$ although remaining acceptable.

Conclusions: Our results suggest that TRUS is equivalent to MR in assessing preBT tumor maximum width in cervical cancer FIGO stage I/II. In more advanced stages TRUS seems to be slightly inferior to MR although maintaining a good agreement to gold standard imaging.

Keywords: Cervical Cancer, Image guided brachytherapy, Ultrasound, TRUS, Magnetic resonance, 3D brachytherapy

\footnotetext{
* Correspondence: mariofedericos@yahoo.it

${ }^{1}$ Radiation Oncology Department, Hospital Universitario de Gran Canaria Dr.

Negrín, Barranco de la Ballena s/n, 35010 Las Palmas de Gran Canaria, Spain

Full list of author information is available at the end of the article
}

(c) The Author(s). 2019 Open Access This article is distributed under the terms of the Creative Commons Attribution 4.0 International License (http://creativecommons.org/licenses/by/4.0/), which permits unrestricted use, distribution, and reproduction in any medium, provided you give appropriate credit to the original author(s) and the source, provide a link to the Creative Commons license, and indicate if changes were made. The Creative Commons Public Domain Dedication waiver (http://creativecommons.org/publicdomain/zero/1.0/) applies to the data made available in this article, unless otherwise stated. 


\section{Background}

Cervical cancer is the fourth most common cancer in women worldwide and the eight overall. A large majority (around $85 \%$ ) of the global burden occurs in the less developed regions [1]. For decades radiotherapy (RT) has been the standard of care for locally advanced disease and brachytherapy (BT) is an essential part of the treatment [2, 3]. In the last decade, 3D treatment planning was introduced for cervical cancer BT [4-9] with outstanding clinical results [10-14]. The goal of $3 \mathrm{D}$ adaptive $\mathrm{BT}$ is to tightly shape the radiation dose to the individual patient's anatomy and tumor topography for each BT fraction, with the in-

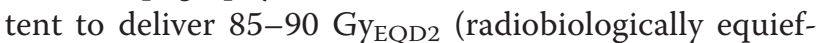
fective dose of $2 \mathrm{~Gy}$ per fraction) to the tumor while minimizing the dose to organs at risk (OAR). The precondition for safe BT treatment individualization is the precise identification of target volumes. Magnetic Resonance (MR) has clear advantages in terms of image quality [15] as it allows the optimal definition of normal peri-cervical soft tissues, tumor extension within the cervix, parametrial infiltration and topography. Additionally, MR enables $4 \mathrm{D}$ volume adaptation following tumor regression during external beam radiotherapy (EBRT) [16, 17]. Unfortunately, due to its costs and limited availability, the majority of patients worldwide are precluded from receiving MRbased BT treatment [18, 19]. Computer tomography (CT) imaging alone is not an alternative to MR because it's poor soft tissue contrast is inadequate to precisely define cervical tumors [20] and burdens of parametrial infiltration [21, 22]. Approaches with less intense MR routine like hybrid MR/CT protocols have been investigated with promising results [23] but still rely on some MR imaging. TRUS has excellent soft tissue resolution, is affordable, and has been used extensively in cervical cancer diagnosis [24]. Moreover, TRUS has been used to aid in proper BT applicator insertion and guidance, and for correct placement of parametrial needles, since it is, among all ultrasound (US) modality, the one that better depicts parametrial infiltration. For all these reasons TRUS is under investigation as a potential alternative to MR for Image-Guided Adaptive Brachytherapy (IGABT) planning [25, 26]. Some shortcomings may however limit TRUS use, such as the intrinsic operator dependency, the inadequate visualization of tumor regression areas in the parametria and lastly, the difficulty in evaluating the relationship of extensive tumors to the pelvic sidewall when the edge of infiltration is beyond the range of the probe. The aim of the present study is a blinded multi-observers comparison of TRUS and MR, assessing tumor maximum width before first BT application (without applicator in place) in a large cohort of cervical cancer patients undergoing IGABT.

\section{Methods \\ Patient characteristics and diagnostic workup}

After ethical committee approval, between 2013 and 2016, 110 consecutive biopsy-proven cervical cancer patients referred to our department were prospectively included. Clinic and pathological features are given in Table 1. Median age was 52.7 year (23.8-88.6) with a vast majority of patients having locally advanced tumors. $54.5 \%$ had tumor width at diagnosis (measured on MR images) larger than $5 \mathrm{~cm}$.

The diagnostic workout consisted of a thorough clinical examination and TRUS imaging acquisition. Clinical findings were reported on a clinical drawing chart. Furthermore, patients underwent thoracic-abdominal contrasted CT and pelvic MR scan. Patients younger than 70 and without obvious macroscopic para-aortic node involvement on $\mathrm{CT} / \mathrm{MR}$ imaging underwent laparoscopic retroperitoneal para-aortic lymphadenectomy [27]. Patients excluded from surgical nodal staging underwent ${ }^{18}$ FDG PET-CT scan.

\section{Treatment}

After CT simulation (Somaton Sensation Open multislice scanner, Siemens), patients received pelvic 3D conformal EBRT (1.8 Gy per fraction up to $45 \mathrm{~Gy}$ total dose), with concomitant chemotherapy (weekly

Table 1 Patient characteristics

\begin{tabular}{|c|c|}
\hline Characteristic & \\
\hline \multicolumn{2}{|l|}{ Age (years) } \\
\hline Median & 52,7 \\
\hline Range & $23.8-88.6$ \\
\hline \multicolumn{2}{|l|}{ Histology (N, \%) } \\
\hline Squamous cell carcinoma & $84(76.3 \%)$ \\
\hline Adenocarcinoma & $19(17.3 \%)$ \\
\hline Adenosquamous cell carcinoma & $5(4.6 \%)$ \\
\hline Clear cell carcinoma & $1(0.9 \%)$ \\
\hline Carcinosarcoma & $1(0.9 \%)$ \\
\hline \multicolumn{2}{|l|}{ FIGO stage $(N, \%)$} \\
\hline $\mathrm{IB}$ & $19(17.3 \%)$ \\
\hline$\| \mathrm{B}$ & $67(60.9 \%)$ \\
\hline$\| \mathrm{IB}$ & $14(12.7 \%)$ \\
\hline IVA & $10(9.1 \%)$ \\
\hline \multicolumn{2}{|l|}{ MR tumor width at diagnosis (N, \%) } \\
\hline$<5 \mathrm{~cm}$ & $50(45.5 \%)$ \\
\hline$>5 \mathrm{~cm}$ & $60(54.5 \%)$ \\
\hline
\end{tabular}

Abbreviations: N Number of patients, FIGO International Federation of Gynaecology and Obstetrics, MR Magnetic Resonance 
intravenous cisplatin, $40 \mathrm{mg} / \mathrm{m}^{2}$ ) when feasible. Paraaortic nodes were included in the RT field in case of histologically proven involvement or positive PET-CT scan.

BT schedule consisted of 4 Ir192 high dose-rate (HDR) intracavitary or intracavitary/interstitial BT fractions of 7 Gy each, within 2 different implants with an interval 7-10 days between insertions. BT insertions were performed under spinal or general anesthesia. BT applicators were MR compatible tandem-ovoids (Utrecht Interstitial CT/MR Applicator, Elekta) or in-house modified MR compatible vaginal cylinder with a perineal template for cases with extended tumor involvement of lower vagina. The interstitial component consisted of plastic needles (ProGuide round tip needles, Elekta) or round tip titanium needles (Elekta). After the first BT insertion patients underwent $1.5 \mathrm{~T}$ MR scan with a phasearray surface pelvic coil (T2 FSE sequences in paraxial, coronal and sagittal plane orientation with a slice thickness of $3.5 \mathrm{~mm}$ ) for applicator reconstruction and dosimetry. The MR scanner was a Magnetom Espree $1.5 \mathrm{~T}$, Siemens. Furthermore, patients underwent a CT scan (2 $\mathrm{mm}$ slice thickness) for research purposes. Images were transferred to Oncentra Brachytherapy planning system (Oncentra Brachytherapy v.4.1, Elekta). Target volumes and OARs were contoured according to GEC-ESTRO recommendations $[4,5]$ and dose optimized to the HRCTV and OARs.

\section{Pre-BT tumor assessment}

One or two days before the first BT insertion, all patients underwent MR scan (preBT MR), which was used for BT application pre-planning. T2 FSE sequences with a phase-array surface pelvic coil (5 $\mathrm{mm}$ slices thickness) in axial and paraxial (adjusting the angle of acquisition to the uterus position in order to obtain a paraxial plane perpendicular to uterine axis), sagittal and coronal orientation were taken with $1 \mathrm{~T}$ (Panorama $1 \mathrm{~T}$ open MR system, Philips Medical System) or $1.5 \mathrm{~T}$ (Siemens Magnetom Espree 1,5 T MRI System) units, depending on the availability. Images were stored on Oncentra Brachytherapy workstation. Furthermore, patients underwent physical examination and clinical features were reported on clinical drawings.

\section{Patients' preparation}

To maximize the quality of TRUS imaging a thorough patient preparation protocol was followed. In details: starting 3 days before the scheduled brachytherapy procedure, all patients were instructed to follow a low fiber and low carb diet; the night before brachytherapy procedure, patients were admitted to the hospital and underwent a bowel preparation protocol consisting of liquid diet and 2 subsequent enemas.

\section{Trans-rectal ultrasound acquisition}

The radiation oncologist in the operating theatre, right before the BT procedure, obtained TRUS images (preBT TRUS) taken with patients under anesthesia, without the applicator in place and according to a standardized protocol. In details: before TRUS image acquisition, a 5/7 F Foley catheter (Histerosonography - Histerosalpingography catheter, Cooper Surgical, USA) was introduced into the uterus in order to visualize cervical canal and the Foley balloon filled with $3-4 \mathrm{cc}$ of sterile saline solution and pulled back up to the uterine internal orifice; bladder was filled with $100 \mathrm{cc}$ of saline solution; bowel preparation was routinely verified as part of the brachytherapy procedure and, in case of poor preparation, a lower bowel irrigation was performed.

The transrectal probe (Hitachi EUP-U533 biplane radial/linear probe $5-10 \mathrm{MHz}$ ) was covered with a lubricated protective sheath, fixed to a ultrasound stepper unit (OncoSelect Stepper, Elekta) and inserted into the rectum. Before image acquisition, the position of transrectal probe was adjusted in order to be parallel to the uterus. This was achieved tilting and rotating freely the probe angle in all directions to overcome recto-sigmoid junction and adjust to the individual patient uterine position in order to maintain the TRUS probe parallel to the uterus. The intrauterine histerosalpingosonography catheter improved the visualization of cervical canal and uterine axis, and helped to define the uterine internal orifice and the transition between upper cervix and lower uterine corpus. Transrectal probe was inserted as much as possible in order to fully visualize the fundus uteri. From this position, a 3D image acquisition was performed with a manual pull-back $(1 \mathrm{~mm}$ step) of the TRUS probe in the stepper unit from the fundus uteri to the lower third of the vagina. The US scanner was a Hitachi EUB 5500. Images were stored and analyzed on Oncentra Prostate workstation (Oncentra Prostate v4.2, Elekta).

\section{Study design, image analysis and measurement procedure}

PreBT MR and preBT TRUS imaging were independently analyzed by three examiners: two radiation oncologists (MF and IR) fully dedicated to brachytherapy, and a US-dedicated radiologist (CRHS). At the time of preBT TRUS imaging analysis, observers were blind to MR images. Moreover, each observer was blind to the other observer's results. First, clinical drawings at diagnosis and at the time of BT were evaluated. With clinical information at hand, preBT TRUS images were analyzed and tumor maximum width measured (HRCTV $\left.\mathrm{HRUS}_{\mathrm{T}}\right)$. Finally, the maximum tumor width was measured on preBT MR $\left(\mathrm{HRCTV}_{\mathrm{MR}}\right)$.

HRCTV $_{\text {TRUS }}$ was defined on gray scale levels as the solid cervical mass, hypoechoic in respect to normal 
parametrium, with eventual continuous extension into parametrial space [24].

HRCTV $_{M R}$ was defined as the macroscopic residual tumor extension visualized on T2 weighted MR, as high signal intensity mass, plus potential surrounding parametrial "grey zones" with intermediate signal intensity in the area of the initial tumor infiltration and the remaining low signal intensity cervical stroma [10]. An example is provided in Fig. 1.

The maximum width measurement was performed as follow: the 3D preBT TRUS imaging was revised and the cranio-caudal axis of the uterus was identified using the cervical canal depicted by the uterosalpingography catheter as reference. The maximum HRCTV $\mathrm{V}_{\text {TRUS }}$ width was measured perpendicular to the uterine axis, along the horizontal transverse axis. The same procedure was followed for the measurement of maximum $\mathrm{HRCTV}_{\mathrm{MR}}$ width.

\section{Statistical analysis}

Descriptive statistics, data analysis, and plots were performed with R Core Team (2014) and Prism 6.0 (2015) software. A Wilcoxon paired $t$-test was used to compare measures. A $p$-value $\leq 0.05$ was considered statistically significant. Intra-class Correlation Coefficient (ICC), Passing-Bablok regression and Bland-Altman plots were used to evaluate the intra- and inter-observers concordance of HRCTV $V_{M R}$ and HRCTV $\mathrm{HRUS}_{\text {T }}$ maximum width measurement.

ICC is a test of concordance or agreement for continuous data and ranges from 0 to 1 [28]. The concept of concordance is that the values obtained in different measurements are identical. With ICC we handle intra- as well inter- observer reproducibility of measurements corresponding to a fixed set of three judges rating each target [29]. In this work an ICC value inferior to 0.4 represent poor agreement, values in between 0.4 and 0.75 represent fair to good agreement and values above 0.75 represent excellent agreement [30].

Passing-Bablok regression method is based on nonparametric model. Result of Passing and Bablok regression consists of several parts and each has its role in interpreting data and concluding on methods agreement. The first result is scatter diagram with regression line that enables visual inspection of measured data and obvious agreement of fitted regression line and identity line. Regression equation $(y=a+b x)$ reveals constant (regression line's intercept (a)) and proportional (regression line's slope (b)) difference with their confidence intervals of $95 \%$ ( $95 \% \mathrm{CI})$. Confidence intervals explain if their value differ from value zero for intercept and value one for slope only by chance. Thus, if $95 \%$ CI for intercept includes value zero it can be concluded that there is no significant difference between obtained intercept value and value zero and there is no constant difference between two methods. Respectively, if 95\% CI for slope includes value one, it can be concluded that there is no significant difference between obtained slope value and value one and there is no proportional difference between two methods. [31].

Bland-Altman residual-like plots are a graphic representation of the data, with the difference between tested measures plotted against their mean values. They define limits of agreement by combining the mean (d) and standard deviations (s) of the differences as $d+2 \mathrm{~s}$ [32].

\section{Results}

One hundred ten consecutive cervical cancer patients were analyzed. HRCTV $\mathrm{HR}_{\mathrm{MR}}$ and $\mathrm{HRCTV}_{\mathrm{TRUS}}$ average measures \pm standard deviations $(\mathrm{SD})$ are given in Table 2.

\section{Intra-observer analysis}

ICC analysis (Table 3) shows an excellent agreement between HRCTV $\mathrm{TRUS}_{\text {ind }}$ and $\mathrm{HRTV}_{\mathrm{MR}}$ maximum width for all three observers. ICC values were slightly lower for larger tumors. Passing-Bablok regression and BlandAltman plots confirm this data (Additional file 1).

Further, a quantitative analysis was undertaken in order to determine the magnitude of the uncertainties between HRCTV $_{M R}$ (considered as gold standard) and HRCTV $_{\text {TRUS }}$ width measurements. Overall average difference between HRCTV $_{M R}$ and HRCTV $_{\text {TRUS }}$ was

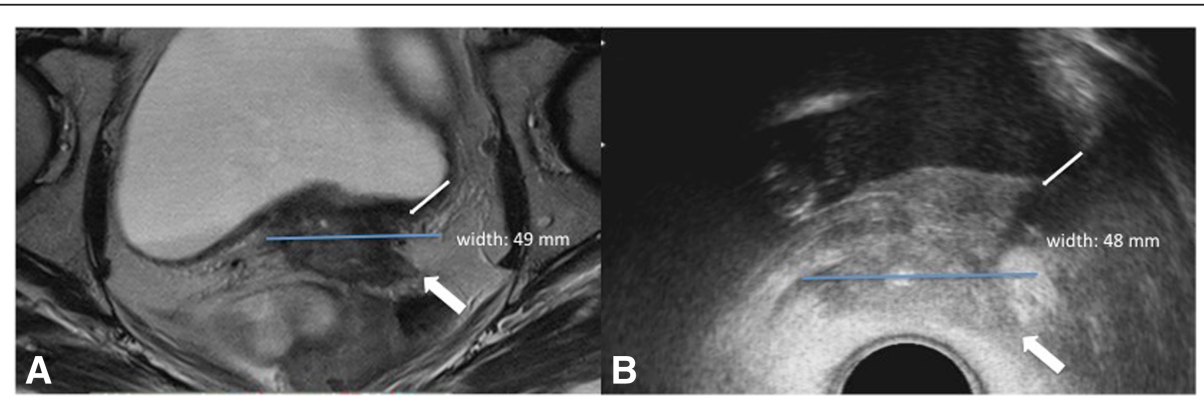

Fig. 1 A case of cervical cancer FIGO stage IVA because bladder infiltration. In preBT MR (a) tumor width is 49 mm. In preBT TRUS (b) tumor width is $48 \mathrm{~mm}$. Thin arrows show posterior bladder wall invasion. Thick arrows show parametrial invasion 
Table 2 Tumor maximum width average measures

\begin{tabular}{|c|c|c|c|c|c|c|}
\hline & \multicolumn{3}{|l|}{$\mathrm{HRCVT}_{\mathrm{MR}}$} & \multicolumn{3}{|l|}{$\mathrm{HRCTV}_{\text {TRUS }}$} \\
\hline & Obs. 1 & Obs. 2 & Obs. 1 & Obs. 2 & Obs. 1 & Obs. 2 \\
\hline All stages & $45.2 \pm 12.7$ & $43.6 \pm 12.6$ & $43.7 \pm 12.2$ & $43.9 \pm 11.4$ & $42.5 \pm 11.9$ & $43 \pm 11.2$ \\
\hline FIGO I & $37.4 \pm 7.7$ & $37.4 \pm 9.2$ & $36.1 \pm 8.8$ & $36.3 \pm 7.3$ & $36.9 \pm 8.7$ & $36.5 \pm 8.4$ \\
\hline $\mathrm{FIGO} \|$ & $41.6 \pm 8.4$ & $39.7 \pm 7.9$ & $40.4 \pm 7.9$ & $40.6 \pm 7.3$ & $39.2 \pm 8$ & $39.6 \pm 6.8$ \\
\hline FIGO III & $60.2 \pm 11.3$ & $58.2 \pm 12.4$ & $58.1 \pm 11.7$ & $57.9 \pm 10.1$ & $54.7 \pm 12.9$ & $57 \pm 10.7$ \\
\hline FIGO IV & $63.7 \pm 11.7$ & $61.5 \pm 11.2$ & $60.1 \pm 11.8$ & $61.2 \pm 9.4$ & $58.3 \pm 12.5$ & $57.9 \pm 11.2$ \\
\hline
\end{tabular}

Tumor maximum width average measures in $\mathrm{mm}$ ( \pm standard deviations) measured in PreBT MR imaging (HRCTV $\mathrm{MR})$ and in PreBT TRUS imaging acquired at the

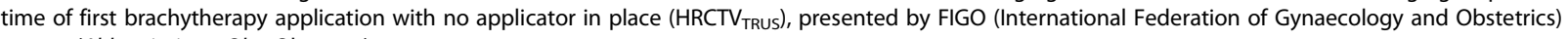
stages. (Abbreviations: Obs. Observer)

$1.3 \pm 3.2 \mathrm{~mm}(p<0.001) ; 1.1 \pm 4.6 \mathrm{~mm}(p=0.01)$ and $0.7 \pm$ $3 \mathrm{~mm}(p=0.01)$ for the three observers, respectively. For FIGO stage I the average difference was $1.1 \pm 2.2$ $\mathrm{mm} \quad(p=0.024), \quad 0.5 \pm 2 \mathrm{~mm} \quad(p=N S)$ and $-0.4 \pm 2.1$ $\mathrm{mm}(p=N S)$. For FIGO stage II it was $1 \pm 2.7 \mathrm{~mm}$ $(p=0.004), 0.5 \pm 1.9 \mathrm{~mm}(p=0.046)$ and $0.7 \pm 3.2 \mathrm{~mm}$ $(p=N S)$. For FIGO stage III the average difference between HRCTV $_{\text {MR }}$ and HRCTV HRUS $_{\text {was }} 2.3 \pm 4.2$ $\mathrm{mm}(p=N S) ; 3.5 \pm 9.6 \mathrm{~mm}(p=N S)$ and $1.1 \pm 2 \mathrm{~mm}$ $(p=N S)$. Finally for FIGO stage IV the difference was $2.5 \pm 5.2 \mathrm{~mm}(p=N S) ; 3.1 \pm 7.3 \mathrm{~mm}(p=N S)$ and $2.2 \pm$ $4 \mathrm{~mm}(p=N S)$ for the three observers respectively.

We defined differences between HRCTV $_{M R}$ and HRCTV $_{\text {TRUS }}$ of less than $3 \mathrm{~mm}$ as minor, between 3 and

Table 3 Intra-observer agreement analysis of tumor maximum width measurements

\begin{tabular}{|c|c|c|c|}
\hline & \multicolumn{3}{|c|}{ HRCVT $T_{M R}$ Vs. HRCTV TRUS } \\
\hline & & ICC & $\mathrm{Cl} 95 \%$ \\
\hline \multirow[t]{3}{*}{ All stages } & Obs. 1 & 0.96 & {$[0.94-0.97]$} \\
\hline & Obs. 2 & 0.93 & {$[0.90-0.95]$} \\
\hline & Obs. 3 & 0.96 & {$[0.95-0.98]$} \\
\hline \multirow[t]{3}{*}{ FIGO I } & Obs. 1 & 0.95 & {$[0.87-0.98]$} \\
\hline & Obs. 2 & 0.98 & [0.94-0.99] \\
\hline & Obs. 3 & 0.97 & [0.92-0.99] \\
\hline \multirow[t]{3}{*}{ FIGO ॥ } & Obs. 1 & 0.94 & {$[0.9-0.96]$} \\
\hline & Obs. 2 & 0.97 & {$[0.95-0.98]$} \\
\hline & Obs. 3 & 0.9 & {$[0.85-0.94]$} \\
\hline \multirow[t]{3}{*}{ FIGO III } & Obs. 1 & 0.91 & {$[0.75-0.97]$} \\
\hline & Obs. 2 & 0.7 & {$[0.3-0.89]$} \\
\hline & Obs. 3 & 0.98 & [0.94-0.99] \\
\hline \multirow[t]{3}{*}{ FIGO IV } & Obs. 1 & 0.87 & {$[0.57-0.96]$} \\
\hline & Obs. 2 & 0.82 & {$[0.44-0.95]$} \\
\hline & Obs. 3 & 0.93 & {$[0.76-0.98]$} \\
\hline \multicolumn{4}{|c|}{$\begin{array}{l}\text { Note that: ICC }>0,75 \text { : excellent agreement; } 0,4-0,75 \text { : fair to good agreement; } \\
\text { < 0,4: poor agreement } \\
\text { Abbreviations: } H R C T V_{M R} \text { High risk clinical target volume maximum width } \\
\text { measured at preBT MR, HRCTV TRUS Tumor maximum width measured at preBT } \\
\text { TRUS, ICC Intra-class Correlation Coefficient, } C \text { C Confidence interval, FIGO }\end{array}$} \\
\hline
\end{tabular}

$5 \mathrm{~mm}$ as potentially relevant and of more than $5 \mathrm{~mm}$ as major.

In the whole cohort of 110 patients (pts), the difference between HRCTV $\mathrm{MR}_{\mathrm{MR}}$ and HRCTV $\mathrm{TRUS}_{\text {TR }}$ measures were less than $3 \mathrm{~mm}$ in 87 pts. (79\%) for observer 1, in 90 pts. (82\%) for observer 2 and in 88 pts. (80\%) for observer 3. It was between 3 and $5 \mathrm{~mm}$ : in 10 pts. (9\%) for observer 1 and 2, and in 11 pts. (10\%) for observer 3 . It was more than $5 \mathrm{~mm}$ in 13 pts. $12 \%$ for observer 1 , in 10 pts. (9\%) for observer 2 and in 11 pts. (10\%) for observer 3 . TRUS was more likely to underestimate tumor width in large FIGO IIIB and IVA tumors (Additional file 1: Figures S2.1 and S2.2).

\section{Inter-observer analysis}

Inter-observer agreement of HRCTV TRUS $_{\text {Hd }}$ $\mathrm{HRCTV}_{\mathrm{MR}}$ maximum width measures was calculated with ICC. As expected, overall agreement of $\mathrm{HRCTV}_{\mathrm{MR}}$ measures was 0.92 (CI95\% 0.89-0.94) with no differences among FIGO stages. Overall HRCTV $\mathrm{HRUS}_{\text {measure }}$ consistency was 0.86 (CI95\% 0.81-0.9). ICC value for HRCTV $_{\text {TRUS }}$ was comparable to HRCTV $_{\text {MR }}$ in FIGO stages I and II tumors and progressively decreased in larger tumors (FIGO stage III and IV), although remaining fairly good (Table 4). In a one-to-one comparison, Passing-Bablok regression and Bland-Altman

Table 4 Inter-observer agreement analysis of tumor maximum width measurements

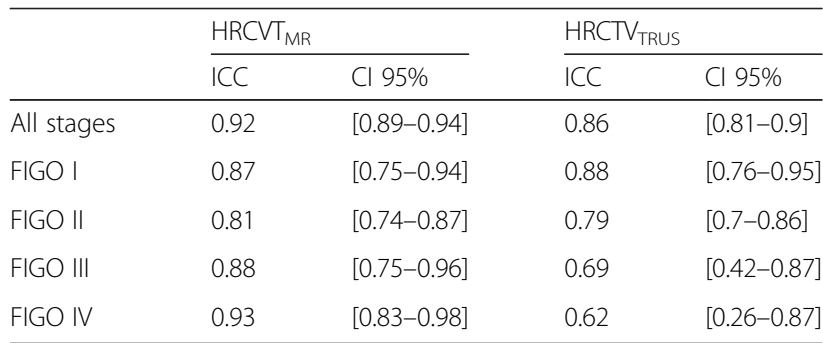

Note that: ICC > 0,75: excellent agreement; 0,4 - 0,75: fair to good agreement; $<0,4$ : poor agreement

Abbreviations: $H R C T V_{M R}$ High risk clinical target volume maximum width measured at preBT MR, HRCTV TRUS, ICC Intra-class Correlation Coefficient, Cl Confidence interval, FIGO International Federation of Gynaecology and Obstetrics 
plots confirmed no substantial differences among individual observers (Additional file 1: Figure S3).

\section{Discussion}

In the last few years US-based BT dose adaptation has been increasingly investigated for cervical cancer IGABT [33]. US has been proven as an excellent diagnostic imaging modality in gynecological oncology [34] and it has been extensively used during BT application to guide tandem and needles insertion [35].

One of the largest prospective studies comparing the diagnostic accuracy of TRUS and MR in the local staging of cervical cancer was published by Fischerova et al. [36] in 2008 and included 95 patients with early-stage disease. The study showed a significantly higher accuracy of TRUS when compared to MR in tumor identification (considering also residual tumor after previous biopsy (93.7 vs. $83.2 \%, p \leq 0.006$ ), or small tumors $\leq 1 \mathrm{~cm}^{3}$ (90.5 vs. $81 \%, p \leq 0.049))$. Similar results were demonstrated by a European multicenter prospective study, which included 182 patients with histologically confirmed earlystage cancer. The diagnostic agreement between ultrasound and pathology was significantly better at detecting residual tumor and parametrial invasion than $\mathrm{MR}(p<$ 0.001). A surprising finding was the maintenance of diagnostic accuracy of ultrasound in the detection of residual tumor after cone biopsy, where it is difficult to distinguish post-inflammatory and reparative changes after the procedure from the presence of residual tumor [37].

Pinkakova et al. in a prospective study on a cohort of 42 FIGO IB1-IIB cervical cancer patients (with limited parametrial involvement) demonstrated the non- inferiority of TRUS with respect to MR in assessing tumor regression during neo-adjuvant chemotherapy [38].

The potential of TRUS imaging in tracking the tumor changes and regression during EBRT is a fact of critical relevance if TRUS is used to guide the BT insertion (normally scheduled after 3-4 weeks of radiochemotherapy) and, potentially, dose adaptation.

The clinical use of US-based BT dose adaptation in cervical cancer BT has been pioneered at Peter MacCallum Cancer Center [39] and promising results have been reported [40]. The method suggested is based on transabdominal US measurements of the uterus taken along the tandem axis in the sagittal plan and has shown a robust correlation with MR measurement. This approach is useful to conform the dose distribution according uterus silhouette in the anteroposterior diameter thus reducing bladder and rectal dose. Nevertheless it seems unfit to target volume delineation at the parametria level, because the limitations of transabdominal US in detecting parametrial invasion and the unfeasibility of true volumetric image acquisition. For all these reasons, Kirisits et al. stated, in an interesting editorial, "this method may be useful, mainly in limited size and well-responding tumors, which are confined to the cervix at the time of BT. However, this clinical scenario does not represent most patients in advanced stage as seen in the countries with high patient numbers and limited resources" [33].

Conversely, TRUS (which is already extensively used for prostate BT imaging and dose optimization) allows a true volumetric image acquisition and a detailed imaging of cervical tumor and eventual extension beyond uterine cervix within the parametrial space [41]. Parametrial infiltration is a well known prognostic parameter for cervical cancer [42] and probably the most relevant factor to take into account at the time of IGABT insertion preplanning [43], to select between intracavitary or intracavitary/interstitial technique [44]. For this reason, the correct assessment of tumor width is a critical point in cervical cancer BT. Validation of TRUS as potentially useful tool for cervical BT imaging and dose adaptation starts from the assessment of the robustness of TRUS measuring tumor width at the time of BT.

Researchers at the Medical University of Vienna in two different studies $[25,45]$ demonstrated an excellent agreement between MR and TRUS in assessing tumor maximum width after EBRT in respectively 16 and 19 patients diagnosed with cervical cancers (FIGO I-IV). In both studies the mean difference between MR and TRUS measurement were in the same range $(-0.3 \pm 3.2 \mathrm{~mm}$ and $-1.1 \pm 3.2 \mathrm{~mm}$ respectively). These data strikingly compare with our results $(1.3 \pm 3.2 \mathrm{~mm} ; 1.1 \pm 4.6 \mathrm{~mm}$ and $0.7 \pm 3 \mathrm{~mm}$ for the 3 observers). We were also able to demonstrate that the magnitude of uncertainty in the vast majority of cases $(\approx 80 \%)$ is very small $(<3 \mathrm{~mm})$, but increases in large tumors. Additionally, we demonstrated that in large IIIB/IVA tumors TRUS may underestimate tumor width compared to MR. Several reasons might be claimed to explain such data.

First of all, the higher degree of uncertainty associated to tumor maximum width measurement in large tumors that spread to the pelvic wall. In fact, even if the maximum tumor width in this study is taken perpendicularly to the cervical canal and uterine axis (in order to improve reproducibility of the measurements between MR and TRUS), the irregular shape of such tumors may impair the identification of a reproducible axis of measurement (Additional file 1: Figure S4). This type of error may be at the basis of the progressively lower TRUS concordance among observers in advanced FIGO stage tumors. On the other hand, such type of uncertainty should be stochastic, while our findings show that TRUS underestimates tumor width ( $>5 \mathrm{~mm}$ ) in $20 \%$ of stage IV and in $7-14 \%$ of stage III tumors (Additional file 1: Figure S2.2). 
A second possible explanation could be an intrinsic limited resolution of TRUS imaging in depicting the limit of tumor infiltration into pelvic wall, because poor image quality (due to patient preparation, or because TRUS shortcoming in evaluating the relationship of extensive tumors to the pelvic sidewall when the edge of infiltration is close to the range of the probe). During this investigation, a strict bowel preparation protocol was followed in order to prevent the interference on TRUS image quality. Nevertheless, TRUS performance in large tumors was inferior to MR, as shown by the inter-observer analysis, where the measurement agreement as expressed by ICC values was extremely high for $\mathrm{HRCTV}_{\mathrm{MR}}$ width, independently from FIGO stage. Conversely, ICC for HRCTV $V_{\text {TRUS }}$ width resulted comparable to MR just for FIGO I/II tumors and progressively declined for larger tumors, although remaining fairly good (Table 4).

A further reason might depend on the shape and type of tumor growth pattern. Very advanced tumor stages (FIGO IVA) show more likely an infiltrative growth with irregular shapes, and thin digitations that deeply infiltrate the parametrial space in comparison to bulky expansive cervical tumors, which are generally more clearly visible in TRUS imaging (Figs. 2 and 3).

It deserves to be mention that a limitation of our study design and a potential bias is that TRUS images were acquired by one investigator only and then analyzed independently by the three investigators. To obtain three sets of TRUS images acquired independently by each observer would be probably more correct. Nevertheless, such study design would be impossible at our Institution because the organization of the workflow. To minimize uncertainties the TRUS acquisition, a protocol (previously described) was thoroughly followed.

Our investigation does not include the assessment of tumor thickness and height. Schmid et al. [25, 45] analyzed tumor thickness as measured by TRUS or MR and found a statistically significant underestimation with TRUS. Such underestimation, in their opinion, is mainly due to the compression of the cervix by the TRUS probe at the moment of image acquisition. We agree with this point. We believe that the insertion of rectal probe (and the angle needed to have it parallel to the uterus) may intrinsically bias any comparison with images taken with relaxed rectum/pelvic floor. On the other hand, the exact burden of tumor cranial infiltration into myometrium (especially after EBRT) is challenging on TRUS imaging (but also on MR). For this reason, in the clinic is generally recommended to load the tandem up to the fundus uteri, making the exact tumor height measurement in MR or TRUS not a critical point.

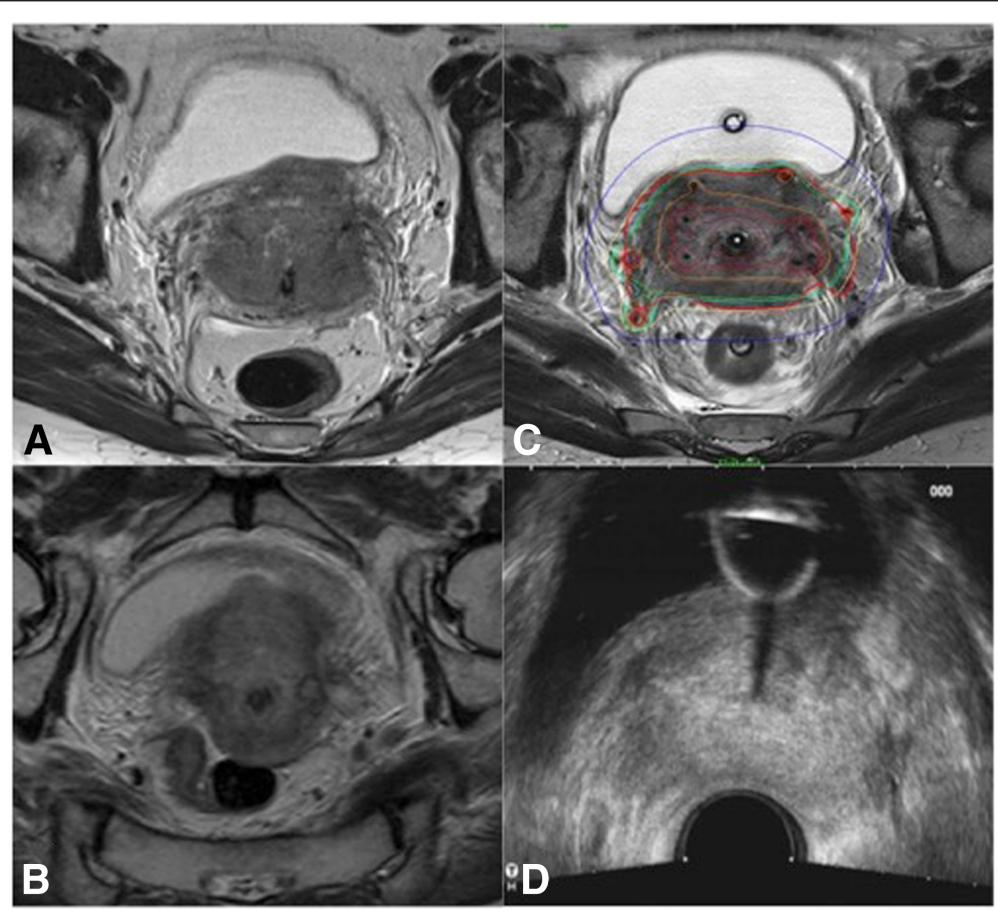

Fig. 2 A case of cervical cancer FIGO stage IVA because bladder infiltration with poor response at EBRT with good agreement between preBT MR and preBT TRUS in tumor measures. Tumor at the time of diagnostic MR (a), at the time of preBT MR (b), at time of first brachytherapy (c) and at time the preBT TRUS (d) 


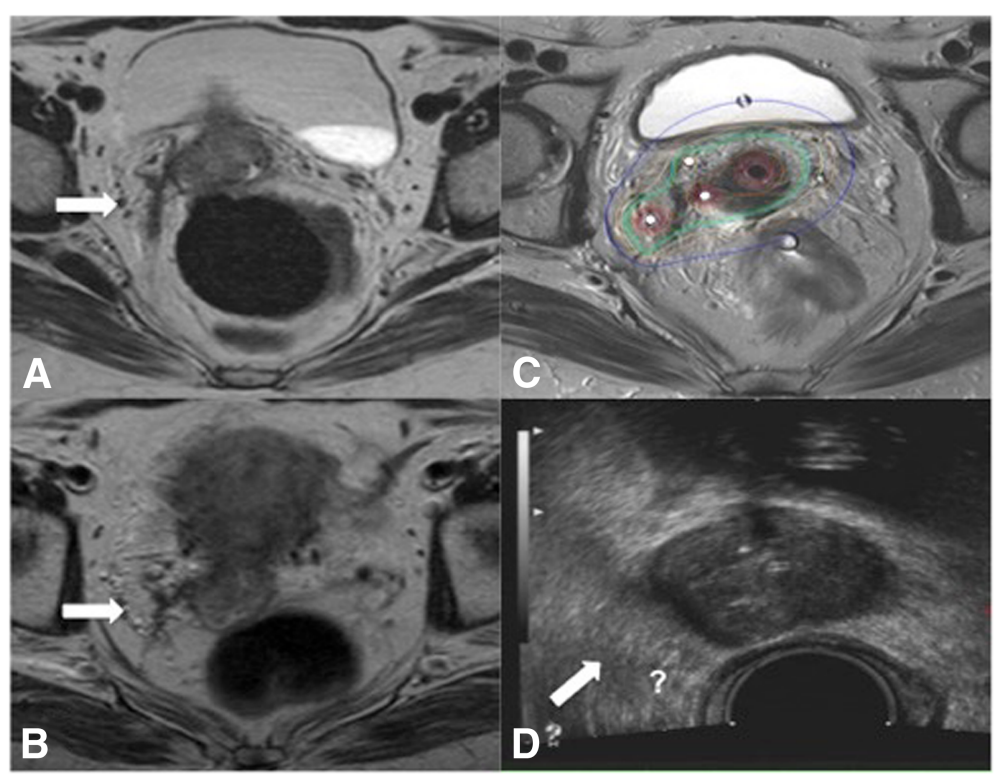

Fig. 3 A case of cervical cancer FIGO stage IIIB with limited response to EBRT with bad agreement between pre BT MR and preBT TRUS in tumor measures. Tumor at the time of diagnostic MR (a), at time of preBT MR (b) and at time of first BT (c). In the preBT TRUS (d) the right parametrial invasion (white arrows) is not as clearly visible as in MR (a-b-c)

\section{Conclusions}

Taken together our results suggest that TRUS is comparable to $\mathrm{MR}$ in assessing preBT tumor maximum width in cervical cancer FIGO stage I/II. In more advance stages TRUS seems to be slightly inferior to MR although maintaining a good agreement to gold standard imaging.

Given the limited cost of TRUS compared to MR and the potential of improved patient accessibility, especially in low income countries, TRUS based IGABT is a major field of research in radiation oncology. Further studies are however still needed to define the technical modality of integration of TRUS in cervical cancer IGABT and TRUS-based dose adaptation.

\section{Additional file}

Additional file 1 (PPT $3891 \mathrm{~kb}$ )

\section{Abbreviations}

BT: Brachytherapy; Cl: Confidence interval; CT: Computer tomography; EBRT: External beam radiotherapy; HRCTV: High risk clinical target volume; ICC: Intra-class correlation coefficient; IGABT: Image guided adapted brachytherapy; MR: Magnetic resonance; OAR: Organs at risk; RT: Radiotherapy; SD: Standard deviation; TRUS: Trans rectal Ultrasound

\section{Acknowledgements}

Authors gratefully acknowledge Dr. Karen S Nkiwane Ph.D for kindly reviewing the manuscript.

\section{Authors' contributions}

MF conceived and designed the study, was responsible of data acquisition, analysis, interpretation and paper writing. CRHS and IR collaborated in the process of data acquisition and analysis and interpretation and paper revision. JGM was primary responsible for data analysis and interpretation. MDRBO helped in data collection, MLSB and PCL participated in data interpretation and paper revision. All authors read and approved the final manuscript.

\section{Funding}

This work was supported by a Fundacion DISA grant \# 019-2016 that allowed to contract a research coordinator.

\section{Availability of data and materials}

The datasets used and analysed during the current study (database with measures) are available from the corresponding author on reasonable request.

Ethics approval and consent to participate

Ethical Committee of the Gran Canaria University Hospital Dr. Negrin approved this study (Protocol Number 2018-001090-24).

\section{Consent for publication}

not applicable.

\section{Competing interests}

The authors declare that they have no competing interests.

\section{Author details}

${ }^{1}$ Radiation Oncology Department, Hospital Universitario de Gran Canaria Dr. Negrín, Barranco de la Ballena s/n, 35010 Las Palmas de Gran Canaria, Spain. ${ }^{2}$ Radiology Department, Hospital Universitario de Gran Canaria Dr. Negrín, Las Palmas de Gran Canaria, Spain. ${ }^{3}$ Unidad de Investigación, Hospital Universitario de Gran Canaria Dr. Negrín, Las Palmas de Gran Canaria, Spain.

Received: 14 March 2019 Accepted: 1 August 2019

Published online: 04 October 2019

\section{References}

1. Ferlay J, Ervik M, Lam F, Colombet M, Mery L, Piñeros M, Znaor A, Soerjomataram I, Bray F. Global Cancer observatory: Cancer today. Lyon: International Agency for Research on Cancer; 2018. Available from: https:/ gco.iarc.fr/today, Accessed 7 July 2019 
2. Lanciano RM, Won M, Coia LR, et al. Pretreatment and treatment factors associated with improved outcome in squamous cell carcinoma of the uterine cervix: a final report of the 1973 and 1978 patterns of care studies. Int J Radiat Oncol Biol Phys. 1991;20:667-76.

3. Han K, Milosevic M, Fyles A, et al. Trends in the utilization of brachytherapy in cervical Cancer in the United States. Int J Radiat Oncol. 2013;87:111-9.

4. Haie-Meder C, Pötter R, Van Limbergen E, et al. Recommendations from Gynaecological (GYN) GEC-ESTRO working group $\boldsymbol{s}^{2}$ (I): concepts and terms in 3D image based 3D treatment planning in cervix cancer brachytherapy with emphasis on MRI assessment of GTV and CTV. Radiother Oncol. 2005; 74:235-45.

5. Pötter R, Haie-Meder C, Van LE, et al. Recommendations from gynaecological (GYN) GEC ESTRO working group (II): concepts and terms in 3D image-based treatment planning in cervix cancer brachytherapy-3D dose volume parameters and aspects of 3D image-based anatomy, radiation physics, radiobiology. Radiother Oncol. 2006;78:67-77.

6. Hellebust TP, Kirisits C, Berger D, et al. Recommendations from Gynaecological (GYN) GEC-ESTRO working group: considerations and pitfalls in commissioning and applicator reconstruction in 3D image-based treatment planning of cervix cancer brachytherapy. Radiother Oncol. 2010; 96:153-60.

7. Dimopoulos JCA, Petrow P, Tanderup K, et al. Recommendations from Gynaecological (GYN) GEC-ESTRO working group (IV): basic principles and parameters for MR imaging within the frame of image based adaptive cervix cancer brachytherapy. Radiother Oncol. 2012;103:113-22.

8. Viswanathan AN, Thomadsen B. American brachytherapy society cervical Cancer recommendations committee, et al. American brachytherapy society consensus guidelines for locally advanced carcinoma of the cervix. Part I: general principles. Brachytherapy. 2012;11:33-46.

9. Viswanathan AN, Beriwal S, De Los Santos JF, et al. American brachytherapy society consensus guidelines for locally advanced carcinoma of the cervix. Part II: high-dose-rate brachytherapy. Brachytherapy. 2012;11:47-52.

10. Pötter R, Georg P, Dimopoulos JCA, et al. Clinical outcome of protocol based image (MRI) guided adaptive brachytherapy combined with 3D conformal radiotherapy with or without chemotherapy in patients with locally advanced cervical cancer. Radiother Oncol. 2011;100:116-23.

11. Ribeiro I, Janssen $H$, De Brabandere $M$, et al. Long term experience with 3D image guided brachytherapy and clinical outcome in cervical cancer patients. Radiother Oncol. 2016;120:447-54.

12. Lindegaard JC, Fokdal LU, Nielsen SK, et al. MRI-guided adaptive radiotherapy in locally advanced cervical cancer from a Nordic perspective. Acta Oncol (Madr). 2013;52:1510-9.

13. Sturdza A, Pötter R, Fokdal LU, et al. Image guided brachytherapy in locally advanced cervical cancer: improved pelvic control and survival in RetroEMBRACE, a multicenter cohort study. Radiother Oncol. 2016;120: 428-33.

14. Charra-Brunaud C, Harter V, Delannes M, et al. Impact of 3D image-based PDR brachytherapy on outcome of patients treated for cervix carcinoma in France: results of the French STIC prospective study. Radiother Oncol. 2012; 103:305-13.

15. Dimopoulos JCA, Schirl G, Baldinger A, et al. MRI assessment of cervical cancer for adaptive radiotherapy. Strahlenther Onkol. 2009;185:282-7.

16. Yoshida K, Jastaniyah N, Sturdza A, et al. Assessment of Parametrial response by growth pattern in patients with International Federation of Gynecology and Obstetrics Stage IIB and IIIB cervical Cancer: analysis of patients from a prospective, multicenter trial (EMBRACE). Int J Radiat Oncol. 2015;93:788-96.

17. Schmid MP, Fidarova E, Pötter R, et al. Magnetic resonance imaging for assessment of parametrial tumour spread and regression patterns in adaptive cervix cancer radiotherapy. Acta Oncol (Madr). 2013;52:1384-90.

18. Grover S, Harkenrider MM, Cho LP, et al. Image guided cervical brachytherapy: 2014 survey of the American brachytherapy society. Int J Radiat Oncol. 2016;94:598-604.

19. Suneja G, Brown D, Chang A, et al. American brachytherapy society: brachytherapy treatment recommendations for locally advanced cervix cancer for low-income and middle-income countries. Brachytherapy. 2017 16:85-94.

20. Hegazy N, Pötter R, Kirisits C, et al. High-risk clinical target volume delineation in CT-guided cervical cancer brachytherapy: impact of information from FIGO stage with or without systematic inclusion of 3D documentation of clinical gynecological examination. Acta Oncol (Madr). 2013;52:1345-52.
21. Pötter R, Federico M, Sturdza A, et al. Value of magnetic resonance imaging without or with applicator in place for target definition in cervix Cancer brachytherapy. Int J Radiat Oncol. 2016;94:588-97.

22. Viswanathan AN, Dimopoulos J, Kirisits C, et al. Computed tomography versus magnetic resonance imaging-based contouring in cervical cancer brachytherapy: results of a prospective trial and preliminary guidelines for standardized contours. Int J Radiat Oncol Biol Phys. 2007;68:491-8

23. Nesvacil N, Pötter R, Sturdza A, et al. Adaptive image guided brachytherapy for cervical cancer: a combined MRI-/CT-planning technique with MRI only at first fraction. Radiother Oncol. 2013;107:75-81.

24. Innocenti P, Pulli F, Savino L, et al. Staging of cervical cancer: reliability of transrectal US. Radiology. 1992;185:201-5.

25. Schmid MP, Nesvacil N, Pötter R, et al. Transrectal ultrasound for imageguided adaptive brachytherapy in cervix cancer - an alternative to MRI for target definition? Radiother Oncol. 2016;120:467-72.

26. Nesvacil N, Schmid MP, Pötter R, et al. Combining transrectal ultrasound and $C T$ for image-guided adaptive brachytherapy of cervical cancer: proof of concept. Brachytherapy. 2016;15:839-44.

27. Benito V, Carballo S, Silva $P$, et al. Should the presence of metastatic Para-aortic lymph nodes in locally advanced cervical Cancer Lead to more aggressive treatment strategies? J Minim Invasive Gynecol. 2017; 24:609-16.

28. Shrout PE, Fleiss JL. Intraclass correlations: uses in assessing rater reliability. Psychol Bull. 1979;86:420-8.

29. Revelle W. Procedures for psychological, psychometric and personality research. Evanston: Northwestern University; 2017.

30. Enderlein G. The design and analysis of clinical experiments. New York. Chichester: Wiley; 1988.

31. Passing $\mathrm{H}$. Bablok. A new biometrical procedure for testing the equality of measurements from two different analytical methods. Application of linear regression procedures for method comparison studies in clinical chemistry, part I. J Clin Chem Clin Biochem. 1983;21:709-20.

32. Bland JM, Altman DG. Statistical methods for assessing agreement between two methods of clinical measurement. Lancet (London, England). 1986;1: 307-10.

33. Kirisits C, Schmid MP, Beriwal S, et al. High-tech image-guided therapy versus low-tech, simple, cheap gynecologic brachytherapy. Brachytherapy. 2015;14:910-2.

34. Fischerova D, Cibula D. Ultrasound in gynecological Cancer: is it time for reevaluation of its uses? Curr Oncol Rep. 2015;17:28.

35. van Dyk S, Schneider M, Kondalsamy-Chennakesavan S, et al. Ultrasound use in gynecologic brachytherapy: time to focus the beam. Brachytherapy. 2015;14:390-400.

36. Fischerova D, Cibula D, Stenhova $H$, et al. Transrectal ultrasound and magnetic resonance imaging in staging of early cervical cancer. Int J Gynecol Cancer. 2008;18:766-72.

37. Epstein E, Testa A, Gaurilcikas A, et al. Early-stage cervical cancer: tumor delineation by magnetic resonance imaging and ultrasound - a European multicenter trial. Gynecol Oncol. 2013;128:449-53.

38. Pinkavova I, Fischerova D, Zikan M, et al. Transrectal ultrasound and magnetic resonance imaging in the evaluation of tumor size following neoadjuvant chemotherapy for locally advanced cervical cancer. Ultrasound Obstet Gynecol. 2013;42:705-12.

39. van Dyk S, Kondalsamy-Chennakesavan S, Schneider M, et al. Comparison of measurements of the uterus and cervix obtained by magnetic resonance and transabdominal ultrasound imaging to identify the brachytherapy target in patients with cervix Cancer. Int J Radiat Oncol. 2014;88:860-5.

40. van Dyk S, Narayan K, Bernshaw D, et al. Clinical outcomes from an innovative protocol using serial ultrasound imaging and a single MR image to guide brachytherapy for locally advanced cervix cancer. Brachytherapy. 2016:15:817-24.

41. Testa AC, Di Legge A, De Blasis I, et al. Imaging techniques for the evaluation of cervical cancer. Best Pract Res Clin Obstet Gynaecol. 2014;28:741-68.

42. Perez CA, Grigsby PW, Chao KS, et al. Tumor size, irradiation dose, and longterm outcome of carcinoma of uterine cervix. Int J Radiat Oncol Biol Phys. 1998:41:307-17.

43. Dimopoulos JCA, Kirisits C, Petric $P$, et al. The Vienna applicator for combined intracavitary and interstitial brachytherapy of cervical cancer: clinical feasibility and preliminary results. Int J Radiat Oncol Biol Phys. 2006; 66:83-90. 
44. Tanderup K, Nielsen SK, Nyvang G-B, et al. From point a to the sculpted pear: MR image guidance significantly improves tumour dose and sparing of organs at risk in brachytherapy of cervical cancer. Radiother Oncol. 2010; 94:173-80.

45. Schmid MP, Pötter R, Brader P, et al. Feasibility of transrectal ultrasonography for assessment of cervical cancer. Strahlenther Onkol. 2013; 189:123-8.

\section{Publisher's Note}

Springer Nature remains neutral with regard to jurisdictional claims in published maps and institutional affiliations.

- fast, convenient online submission

- thorough peer review by experienced researchers in your field

- rapid publication on acceptance

- support for research data, including large and complex data types

- gold Open Access which fosters wider collaboration and increased citations

- maximum visibility for your research: over $100 \mathrm{M}$ website views per year

At $\mathrm{BMC}$, research is always in progress. 